\title{
La respuesta de prevención como política de salud pública del Estado peruano en el caso de propagación internacional de enfermedades. Un análisis a propósito del COVID-19
}

\author{
Guisseppi Paul Morales Cauti ${ }^{1}$ \\ Universidad Privada Norbert Wiener \\ guisseppi.morales@uwiener.edu.pe \\ Código ORCID: https://orcid.org/0000-0002-6550-0722 \\ Adriana Abril Camones Alcazar ${ }^{2}$ \\ Universidad Privada Norbert Wiener \\ alcazaradriana@gmail.com \\ Código ORCID: https://orcid.org/0000-0002-8597-5610
}

\section{Resumen:}

En la presente investigación se analiza el desarrollo de las políticas públicas a cargo del Estado peruano en materia de prevención de propagación internacional de enfermedades a propósito de la pandemia provocada por el COVID-19. La estrategia de análisis parte de la revisión sistemática de las disposiciones normativas dada por la Organización Mundial de la Salud -especialmente las dictadas mediante el Reglamento Sanitario Internacional-, la Organización panamericana de la Salud y las entidades del Estado peruano encargadas de desarrollar e implementar las políticas públicas de respuesta de prevención y control de la propagación del virus SARS-CoV-2 (COVID-19). Se obtuvo como resultado que el Estado peruano no diseñó ni implementó las políticas públicas como respuesta de prevención en el caso de propagación internacional de enfermedades, conforme lo había exigido la Organización Mundial de la Salud y la Organización Panamericana de la Salud, en documentos oficiales de cumplimiento obligatorio para el Estado peruano. Esta situación ha generado consecuencias negativas posteriores en el contexto de la propagación internacional de la enfermedad del COVID -19 provocando la muerte de ciudadanos peruanos que pudieron evitarse; a tal punto que el Perú es el país con mayor mortalidad en el mundo en el contexto de esta pandemia.

Palabras clave: Prevención de enfermedades, política pública, propagación internacional, pandemia, COVID-19. 


\section{Abstract:}

This research analyses the development of public policies by the Peruvian State on the prevention of international spread of diseases in the pandemic caused by the COVID-19. The analysis strategy is based on the systematic review of the regulatory provisions given by the World Health Organization - especially those issued through the International Health Regulations - by the Pan American Health Organization and the Peruvian State entities responsible for developing and implementing public policies for the prevention and control of the spread of SARS-CoV-2 virus (COVID-19). It was obtained as a result that the Peruvian State did not design or implement public policies in the case of international disease spread, as required by the World Health Organization and the Pan American Health Organization, in official mandatory compliance documents for the Peruvian State. This situation has generated subsequent negative consequences in the context of the international spread of Covid-19 disease causing the death of Peruvian citizens that could be avoided; to such an extent that Peru is the country with the highest mortality in the world in the context of this pandemic.

Key words: Disease prevention, public policy, international spread, pandemic, COVID-19. 


\section{Realidad problemática}

El Estado peruano es parte de la comunidad jurídica internacional en diferentes ámbitos, incluida aquella que tiene como función la regulación de la salud a nivel mundial y regional a cargo de los órganos rectores -la Organización Mundial de la Salud (OMS) y la Organización Panamericana de la Salud (OPS) - respectivamente. En consecuencia, las políticas en materia de salud pública propuestas desde estos dos organismos internacionales son vinculantes para el Estado peruano y, por lo mismo, este asume la obligación de observar e implementarla. Así lo establece el artículo $2^{\circ}$ a) de la Constitución de la OMS -aprobada en 1946 y cuya entrada en vigor se remonta a 1948- cuando prescribe que se constituye como "autoridad directiva y coordinadora en asuntos de sanidad internacional". Esta situación se justifica en la medida que la salud humana requiere del conjunto de la sociedad organizada para dedicarse, con prioridad, a prevenir la enfermedad y promover la salud (Rojas, 2004). En efecto, la sociedad mundial cuenta con una organización especializada y jerarquizada que tiene como finalidad la atención de la salud pública mundial como es el caso de la prevención y control de propagación internacional de enfermedades.

En este contexto, se viene experimentando, a partir de diciembre del año 2019, uno de los eventos de mayor impacto en materia de sanidad internacional, como es la pandemia a raíz de la propagación del virus conocido como SARS-CoV-2 que provoca la enfermedad del COVID-19, el cual, desde la lejana ciudad de Wuhan en China, ha puesto en vilo incluso a las poblaciones más apartadas de las grandes metrópolis, tanto así que ha infectado a más de 113 millones y provocado la muerte de 2,420,550 personas en todo el mundo, hasta el 28 de febrero de 2021 .

Sin embargo, la materia controvertida -para los fines de esta reflexión-, no se circunscribe al impacto que viene causando la propagación del mencionado virus, sino al análisis respecto del diseño e implementación de una política pública como respuesta de prevención de propagación internacional de enfermedades a cargo del Estado peruano, en el marco de las directivas emitidas por la OMS y de la OPS.

En la medida que el Perú haya incurrido en omisiones o defectos en el diseño o implementación de dichas políticas públicas, este análisis podrá servir de fundamento para explicar el impacto que viene generando la propagación del COVID-19 en territorio peruano. En ese sentido, la investigación que se propone adquiere relevancia en la medida que presentará una sistematización de datos desde la perspectiva de la adopción e implementación de políticas públicas de prevención de propagación internacional de enfermedades, como responsabilidad del Estado peruano, conforme a las directivas de la OMS y a la función estatal en materia de salud pública que le corresponde, conforme al artículo $9^{\circ}$ de la Constitución Política del Perú.

\section{La respuesta de prevención y el control de enfermedades en el marco del comercio internacional y el tráfico mundial de personas}

La OMS en el Informe Sobre la Salud en el Mundo (Ginebra, 2007) declaró que, desde el año 1967, se habían detectado "al menos 39 agentes patógenos nuevos, entre ellos el VIH y los causantes de la fiebre hemorrágica del Ébola, la fiebre de Marburgo y el SRAS" (s/p). Éstas y otras enfermedades -entre ellas las gripes pandémicas- constituían una permanente amenaza a la salud mundial. Debido a este peligro latente, y, con la finalidad de diseñar e implementar estrategias coordinadas de alcance mundial, la OMS recomendó la plena entrada en vigencia del Reglamento Sanitario Internacional (RSI), dado que, desde 1969 -fecha de su adopción-, había carecido de eficacia. Este problema se subsanó a partir de 23 de mayo de 2005, y desde aquel tiempo las medidas sanitarias adoptarían vigor a fin de diseñar e implementar las políticas públicas orientadas a prevenir la propagación internacional de enfermedades o la contaminación. (OMS-RSI, p. 8). 
La prevención y control de enfermedades a partir de este año fue convirtiéndose en un asunto de atención, no solo necesaria, sino principalmente urgente, en la medida que se incrementaba significativamente el tráfico internacional, el cual se entiende, en términos de la OMS (RSI), como "el movimiento de viajeros, equipajes, cargas, contenedores, medios de transporte, mercancías o paquetes postales a través de una frontera internacional, con inclusión del comercio internacional" (p.10). Con la suscripción de múltiples tratados internacionales se liberalizaron aún más las transacciones internacionales, promovidas por la Organización Mundial del Comercio (OMC). En este escenario de las relaciones internacionales, la OMS y la OPS asumieron, con conciencia crítica, la imperiosa necesidad de adoptar medidas sanitarias que garanticen el cumplimiento de la finalidad y alcance del RSI, el cual consiste en "(...) prevenir la propagación internacional de enfermedades, proteger contra esa propagación, controlarla y darle una respuesta de salud pública proporcionada y restringida a los riesgos para la salud pública y evitando al mismo tiempo las interferencias innecesarias con el tráfico y el comercio internacionales" (art. 2).

La OMS asume y reconoce que el comercio internacional, tal como está diseñada la economía mundial, es un fenómeno inevitable. En ese marco, la OMC y la OMS suscribieron acuerdos para garantizar la continuidad del comercio internacional en un escenario de permanente amenaza a la salud mundial como consecuencia del riesgo de la propagación de enfermedades y, a la vez, evitar la adopción de medidas extremas, como la cuarentena o el embargo comercial, que pudieran perturbar, obstaculizar o impedir el comercio mundial. La continuidad del comercio internacional puede garantizarse mediante la aplicación de "sistemas de vigilancia de alerta temprana, planes de preparación para epidemias, creación de reservas de medicamentos esenciales, agilización de las comunicaciones, y difusión de toda la información disponible a través de redes, con el fin de facilitar una pronta intervención" (OMS-OMC, 2002, p. 13). Esta alianza se hace necesaria en el marco de una política de salud y de comercio internacional coherentes, compatibles y que, además, produzcan recíproco beneficio para ambos sectores en la medida que constituyen sectores claves para la construcción de un mundo con desarrollo económico, pero con bienestar integral para la persona sobre la base de la inversión generadora de empleo.

Por ello, la aplicación del artículo $13^{\circ}$ del RSI se hizo cada vez más necesaria y, el compromiso de los Estados Parte, más urgente. La adopción de medidas de prevención, protección y control de la salud pública con la finalidad de dar respuesta oportuna y eficaz ante eventuales escenarios de propagación internacional de enfermedades, no puede tener éxito sin el diseño e implementación de políticas coherentes y eficaces de alcance mundial, con intervención de todos los actores involucrados. Si se deseaba alcanzar los objetivos propuestos, las grandes directrices emanadas por los órganos rectores en materia de salud regional y mundial debieron tener su respectiva implementación conforme al compromiso y responsabilidad de los Estados.

Por otro lado, mediante el Informe sobre la salud en el mundo (2007), la OMS resalta que las amenazas a la salud mundial son cada vez más acuciantes debido a la mayor interconexión entre los países y, con ello, las enfermedades que aparecen con cierta frecuencia tienen una velocidad de propagación a un ritmo cada vez mayor. Esta situación no podría ser de otra manera si se toma en cuenta que en el año 2006 viajaron en avión 2100 millones de pasajeros en el mundo y en el año 2007 se movilizaron por el mundo más de 2000 millones de personas (OMS, 2007). En el año 2019, según la Organización Mundial del Turismo (OMT) el tráfico mundial de personas -sin considerar los viajes internacionales por otras razones- había experimentado la llegada de 220 millones de turistas para América, 745 millones para Europa, 73 millones para África, 61 millones para Oriente Medio y 361 millones para Asia y el Pacífico (OMT, 2019). Con este nivel de tráfico de personas el brote o epidemia en cierto lugar del mundo puede expandirse a cualquier lugar del planeta en muy corto tiempo, por lo que el diseño e implementación de políticas integrales de prevención y control de propagación de enfermedades adquiere un carácter de necesidad pública de primer orden. 
Atendiendo a lo expuesto, el Dr. Tedros Adhanom Ghebreyesus, Director General de la OMS, declaró como "emergencia de salud pública de importancia internacional" al brote del coronavirus (COVID-19). Asimismo, estableció una necesaria cooperación entre la OMS y la OMT con la finalidad de mitigar los efectos de la pandemia en el sector turismo. Las medidas preventivas adoptadas pasan por la "detección y gestión de los casos sospechosos en los puntos de entrada, en particular los puertos, aeropuertos y pasos fronterizos terrestres" (OMS, 2020, p. 2). Además, conscientes de que uno de los mayores problemas que afecta a la humanidad es el acceso a los medicamentos, sobre todo en las poblaciones más pobres del mundo, la OMC y la OMS han establecido criterios de ayuda recíproca en materia de abastecimiento de medicinas, como es el caso de la posibilidad de eliminar o reducir los aranceles para su importación.

\section{La salud pública: responsabilidad de la Organización Mundial de la Salud (OMS) y de los Estados parte}

La salud pública, entendida no solo como la ausencia de enfermedad, sino como el estado de bienestar somático, psicológico y social del individuo y de la colectividad, conforme lo ha establecido la OMS, no puede ser atendida sino mediante una adecuada coordinación entre las entidades especializadas desde los niveles de mayor relevancia a nivel mundial hasta las instituciones encargadas de aplicar las acciones más concretas en cada uno de los Estados. Este nivel de organización exige no solo la creación y el reconocimiento -o legitimidad- de los órganos encargados de diseñar las políticas de atención a la salud, sino también la generación de instrumentos adecuados que viabilicen su aplicación, en el nivel de la prevención y control, y, en este caso, de la propagación internacional de enfermedades. Pero también implica el compromiso de los Estados, como condición ineludible, para establecer estrategias en el marco de la respuesta eficaz en materia de prevención y control de propagación de enfermedades.

En ese sentido, Gómez (2020, p. 7) ha sostenido que la OMS en 1949 tuvo la necesidad de homologar la salud al bienestar y esto "(...) se constituyó en el soporte formal de las políticas públicas que orientaron por muchos años la gestión sanitaria en casi todo el mundo". Desde entonces, la OMS, en concordancia con los Estados miembros, tiene la responsabilidad de diseñar e implementar las políticas para garantizar el disfrute del derecho a la salud de las personas. Parte de esta responsabilidad recae en el compromiso de desarrollar e implementar políticas de prevención y control de enfermedades, entendidas como acciones de gobierno orientadas a la satisfacción de algún interés o necesidad pública, para enfrentar los problemas sanitarios prioritarios mediante actividades transversales de prevención y promoción de la salud.

Para el cumplimiento de estos fines, la OMS promovió la aprobación del Reglamento Sanitario Internacional (RSI) adoptado en 1969 pero sin mayor eficacia, salvo luego de su revisión que fuera adoptado por la 58 $8^{\circ}$ Asamblea Mundial de la Salud, el 23 de mayo de 2005. Nunca antes la salud pública mundial había sido materia de especial preocupación, sino hasta la entrada en vigencia del RSI que en su art. 3.3 propone la "(...) protección de todos los pueblos del mundo frente a la propagación internacional de enfermedades". Pero esta labor tuitiva en materia de salud pública no podría concretarse si no es con la participación decidida y orgánica de los Estados parte. De allí que el art. 13.1 del RSI prescriba lo siguiente:

"Cada Estado Parte desarrollará, reforzará y mantendrá, lo antes posible, pero a más tardar cinco años después de la fecha de entrada en vigor del presente Reglamento para ese Estado Parte, la capacidad necesaria para responder con prontitud y eficacia a los riesgos para la salud pública y las emergencias de salud pública de importancia internacional (...)”.

Como puede advertirse, el compromiso del Estado peruano frente a este marco normativo internacional es desarrollar las políticas públicas a fin de cumplir con la finalidad propuesta, más aún si el RSI para el Perú, así como para otros países, entró en vigor a partir del 15 de junio de 2007, conforme a la relación de países que se encuentra en el apéndice 1 del RSI. A partir de entonces, el 
Perú asumió la obligación de implementar las disposiciones de la OMS en materia de prevención y control de propagación internacional de enfermedades, además de atender las recomendaciones formuladas, con la finalidad de garantizar un nivel adecuado de seguridad sanitaria.

En el Informe sobre la salud en el mundo (2007), la OMS alertó sobre la necesidad de fortalecer los sistemas sanitarios; asimismo, dio cuenta de que, en las anteriores experiencias, las emergencias sanitarias pudieron haberse prevenido si los Estados hubieran cumplido oportunamente con implementar con pertinencia su sistema sanitario.

En consecuencia, para hacer frente a una emergencia sanitaria de impacto internacional que afecte la salud pública más allá de las fronteras de un Estado -lo que la OMS ha denominado "emergencia de salud pública de importancia internacional" que amerita además una respuesta internacional coordinada" (RSI, p.7, 2008)-, se han establecido estrategias y metas concretas que pasan por una necesidad ineludible de coordinación permanente entre Estados, dado que no será posible afrontar de forma aislada o individual un problema de esta naturaleza. En ese sentido, en el referido informe se han formulado recomendaciones estratégicas que los Estados debieron implementar progresivamente para el cumplimiento de esta finalidad, tales como:

a. Fortalecer y mantener la capacidad para detectar (vigilar), notificar y responder rápidamente a los eventos de salud pública, entendidos como "la manifestación de una enfermedad o un suceso potencialmente patógeno" (p. 7, 2008);

b. La responsabilidad de fortalecer la capacidad de la infraestructura de salud pública ( $p$. 5, 2008);

c. Dotar de mayores recursos para la formación, vigilancia, capacidad de laboratorio, redes de respuesta y campañas de prevención (p.71, 2008).

En el Informe sobre la salud en el mundo (2008), la OMS recomendó la aplicación de políticas sistémicas y políticas de salud pública orientadas a garantizar, por un lado, el acceso universal y la prestación eficaz de los servicios de salud y, por otro lado, hacer frente a los problemas sanitarios con eficacia, a través de la prevención y promoción de la salud. Para la OMS, las metas de atención de la salud podrán alcanzarse con mayor eficacia mediante la implementación de lo que ha denominado "la atención primaria de la salud". Esta puede entenderse como una estrategia de organización integral que tiene como propósito la cobertura universal a la salud, la garantía de una respuesta de salud con calidad, equidad, solidaridad, participación social y rendición de cuentas del gobierno (Macinko, Montenegro, Nebot y Etienne, 2007).

Para concretar una atención primaria deben desarrollarse políticas públicas eficaces que impliquen la participación desde niveles inferiores en el Estado, hasta la coordinación supranacional, como única estrategia que procure un adecuado funcionamiento de estas políticas (OMS, 2008). Estas deben desarrollarse en los siguientes ámbitos:

a. Respecto de los sistemas sanitarios, estos deben estar orientados a garantizar el acceso a los medicamentos esenciales, recursos humanos suficientes, en control de calidad, entre otros aspectos.

b. En el ámbito de las políticas públicas eficaces, debe atenderse la intervención estatal en materia de higiene pública, prevención de enfermedades, la capacidad de respuesta rápida para hacer frente a epidemias y catástrofes.

c. Las vinculadas a mejorar la salud desde sectores distintos del sanitario, como es el caso de la educación de los escolares y la seguridad alimenticia como factores que influyen significativamente en la prevención de enfermedades. 


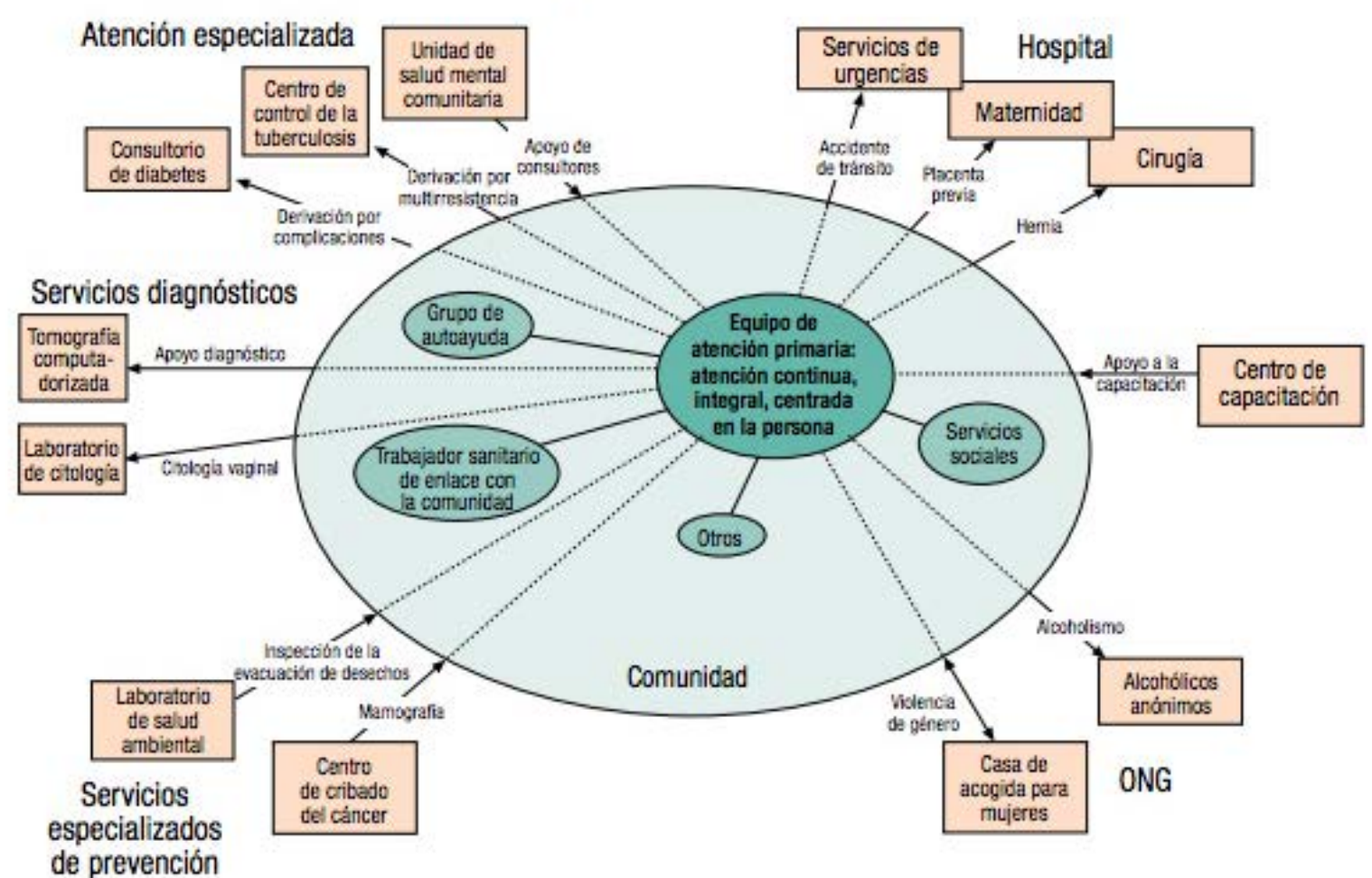

Figura 1. Sistema Integrado de Atención Primaria en Salud (OMS, 2008, p. 59).

\section{La Organización Panamericana de la Salud y las políticas públicas como respuesta de prevención de propagación internacional de enfermedades}

La Organización Panamericana de la Salud (OPS) también es parte de esta preocupación por diseñar y coordinar la aplicación de políticas públicas preventivas de propagación internacional de enfermedades. En ese sentido, en el año 2007 se aprobó en la ciudad de Panamá, la Agenda de Salud para las Américas 2008-2017, en cuyo análisis situacional de la salud en la región ya estaba pronosticado la posibilidad de sufrir graves afectaciones a la salud regional. Así, se sostuvo que "Entre las enfermedades transmisibles persisten las amenazas tradicionales, como la malaria, pero han aparecido nuevas amenazas (...) y se han producido cambios en las características de algunos agentes, tales como los virus de la influenza, cuyas variantes podrían provocar una pandemia de graves consecuencias" (p. 7, 2008).

Al establecer las áreas de acción, la OPS sugirió expresamente el fortalecimiento de los sistemas de vigilancia epidemiológica que tome en cuenta, entre otros factores, los estilos de vida como medida preventiva de la salud. Asimismo, hizo especial énfasis en la necesidad de diseñar estrategias para la atención primaria de la salud, el acceso equitativo y universal a los servicios de salud que brinda el Estado -con especial énfasis en las zonas rurales de la sociedad- y la mejora de los sistemas de información sanitaria (La Organización Panamericana de la Salud, 2007, p. 15).

En suma, la OPS (2007, p. 21) había advertido a los Estados de América, con suficiente claridad, para que puedan "(...) prepararse y tomar medidas intersectoriales para enfrentar desastres, pandemias y enfermedades que afectan la seguridad sanitaria nacional, regional y global", y 
recomendó que asuman nuevas obligaciones establecidas en el RSI para prevenir y controlar la propagación de enfermedades tanto al interior como fuera de sus fronteras. Para este fin planteó la necesidad de

a) Fortalecer el proceso efectivo de integración subregional, regional y global.

b) Fortalecer el trabajo conjunto con el área de ganadería y agricultura para la prevención de enfermedades zoonóticas.

c) Fortalecer las capacidades de salud pública.

En el ámbito de la salud, la prevención, en términos de Redondo (2004), se entiende como la acción que normalmente emana desde los servicios de salud y que considera a los individuos y a las poblaciones como expuestas a factores de riesgo identificables, que suelen ser con frecuencia asociados a diferentes conductas de riesgo de los individuos. La modificación de estas conductas de riesgo constituye una de las metas primordiales de la prevención de la enfermedad (Redondo, 2004, p. 8).

Es este contexto, de constante preocupación por la salud regional que la OPS, en el que, mediante el Módulo de Principios de Epidemiología para el Control de Enfermedades (2011), se propone 4 niveles de prevención, según el grado de desarrollo de la enfermedad: la prevención primordial, dirigida a evitar el surgimiento de patrones de vida que contribuyen a elevar el riesgo a enfermar (como el consumo de alimentos alto en grasas o azúcares); la prevención primaria, orientada a mitigar los efectos de la enfermedad a través del control de sus causa y sus factores de riesgo (como la cloración del agua, la pasteurización de la leche); la prevención secundaria, referida a la curación y a la mitigación de las consecuencias mediante la atención temprana de enfermedades, procurando reducir la letalidad de la enfermedad (el tratamiento temprano del cáncer es el ejemplo típico de este tipo de prevención; y la prevención terciaria, orientada a reducir los efectos de una enfermedad a través de una atención médica adecuada y oportuna.

Para darle continuidad a los objetivos de políticas públicas en materia de salud plasmadas en la Agenda de Salud para las Américas 2008-2017, la OPS (2017) -con la finalidad de garantizar una salud más equitativa como vehículo para generar bienestar en la población-, aprobó la "Agenda de salud sostenible para las Américas 2018-2030", estableciendo 11 objetivos estratégicos, de los cuales, en esta oportunidad, solo se citarán los pertinentes en el marco de esta investigación:

Objetivo 1. Ampliar el acceso equitativo a servicios de salud integrales, integrados y de calidad centrados en la persona, la familia y la comunidad, con énfasis en la promoción de la salud y la prevención de las enfermedades. Este objetivo implica el compromiso de los Estados de generar condiciones que permitan un acceso universal a la salud y cobertura universal de la salud, así como la implementación de modelos de atención de la salud con capacidad resolutiva en el primer nivel de atención.

Objetivo 4. Asegurar el financiamiento adecuado y sostenible de la salud, con equidad y eficiencia, y avanzar hacia la protección contra los riesgos financieros para todas las personas y sus familias. Este objetivo implica el compromiso de los Estados de incrementar el gasto público en equipamiento, capacitación del personal, mejoramiento de la infraestructura, entre otros.

Objetivo 5. Asegurar el acceso a medicamentos esenciales y vacunas de manera oportuna.

Objetivo 8. Fortalecer la capacidad nacional y regional de preparación, prevención, detección, vigilancia y respuesta a los brotes de enfermedades y a las emergencias y los desastres que afectan la salud de la población. Este objetivo busca reducir la mortalidad y morbilidad 
mediante mecanismos de prevención de agentes patógenos, establecer estrategias de prevención, alerta y control de peligros infecciosos.

Si bien cada uno de los objetivos propuestos por la OPS resultan trascendentes, es meritorio resaltar la interrelación que guardan entre sí. En consecuencia, el logro de un objetivo genera condiciones favorables para el logro de los demás. En ese sentido, el fortalecimiento de la capacidad de prevención, vigilancia y respuesta de los brotes de enfermedades está condicionado por el logro de otros objetivos propuestos en esta agenda de salud.

Aun cuando las recomendaciones tanto de la OMS y de la OPS versan sobre distintos aspectos de la salud pública, como el referido a la gestión desde el Estado, acceso a la salud, desigualdades en materia de materia de acceso a la salud, control de enfermedades no transmisibles, etc. En esta oportunidad se ha procurado resaltar aquello que tiene relación directa con las políticas de respuesta de prevención en el contexto de la propagación internacional de enfermedades.

\section{Marco normativo como fundamento para la respuesta del Estado peruano en materia de prevención de propagación internacional de enfermedades}

De acuerdo con el artículo $9^{\circ}$ de la Constitución Política del Perú (1993), el Estado asume la responsabilidad de determinar la política nacional de salud, desde la fase del diseño, ejecución, control y evaluación. Esta responsabilidad tiene sustento en la medida que pretende dotar de eficacia a lo dispuesto en el artículo $7^{\circ}$ de la misma carta magna, que consagra el derecho fundamental a la salud, en favor de todos los ciudadanos. Pero también debe entenderse en el contexto de lo dispuesto en el artículo $43^{\circ}$ de la ley fundamental en el que se reconoce al Perú, como un Estado Social porque "no solo reconoce, protege y promueve el goce de derechos tales como la libertad, la seguridad y la igualdad (...) si no que pretende conseguir su mayor efectividad" (García, 2013, p. 942) las que solo serán posibles si el Estado asume acciones positivas, como el diseño e implementación de políticas públicas en materia de salud, como uno de los principales ámbitos de protección de la persona humana.

Es objeto de debate el rol que ha desempeñado el Estado peruano en el marco de la Constitución de 1993. Se cuestiona su inclinación por el diseño e implementación de políticas liberales clásicas, sobre todo en materia económica, lo que habría significado una excesiva flexibilización del rol garante de la satisfacción de las necesidades esenciales de la población. Esta situación mantiene un vínculo estrecho con la regulación contenida en el art. $58^{\circ}$ de la Constitución Política referida al régimen económico que promueve la iniciativa privada ejercida en el marco de la economía social de mercado. Asimismo, se dispone que el Estado debe desempeñar su rol fundamentalmente promotor de la inversión privada y eximirse de participar como agente económico, salvo excepciones (art. 60). Importa tomar en consideración el modelo económico que se viene implementado en el Perú porque la satisfacción de las necesidades básicas no tiene la misma eficacia si estas son atendidas directamente por el Estado o mediante la participación de la empresa privada. A menudo se cree que la empresa privada garantiza la eficiencia en la prestación de servicios, pero esta no está exenta de problemas, como se ha experimentado en el Perú durante todo el periodo de vigencia de la Constitución de 1993. Un factor que afecta gravemente la implementación de políticas públicas en el marco de la transparencia y la eficiencia es la corrupción en la empresa privada. Es sintomático en el Perú que la corrupción genere una pérdida de $4 \%$ a $5 \%$ del PBI (Medina, 2020), lo que representó aproximadamente 23,000 millones de soles en el año 2019, correspondiente a la ejecución neta del presupuesto anual (Shack, 2020).

Por otro lado, los artículos constitucionales $7^{\circ}$ (Derecho a la salud) y $9^{\circ}$ (Política Nacional de Salud), han merecido un desarrollo legislativo mediante la Ley $\mathrm{N}^{\circ} 26842$, Ley General de Salud, (promulgada el 15 de julio de 1997) en cuyo artículo IV del TP se prescribe que "la salud pública es responsabilidad primaria del Estado (...)". 
Asimismo, mediante Decreto Legislativo $N^{\circ} 1161$ (publicado el 7 de diciembre de 2013), que aprueba la Ley de Organización y Funciones del Ministerio de Salud, se dispone que el Estado peruano, a través del Ministerio de Salud, asume la función rectora de formular, planear, dirigir, coordinar, ejecutar, supervisar y evaluar la política nacional y sectorial de promoción de la Salud, la prevención de enfermedades, recuperación y rehabilitación en salud (...)" (art.5, a). En el artículo $76^{\circ}$ de la Ley $\mathrm{N}^{\circ} 26842$, se dispone que la autoridad de salud es responsable de dirigir y normar las acciones destinadas a evitar la propagación y lograr el control y erradicación de las enfermedades transmisibles en todo el territorio nacional, en tanto ejerce la vigilancia epidemiológica e inteligencia sanitaria y dictando las disposiciones correspondientes.

Finalmente, mediante D.S. № 025-2005-SA que aprueba el Reglamento de Organización y Funciones del Ministerio de Salud, se asigna la función de velar por el cumplimiento de las disposiciones del RSI a la Dirección de Vigilancia Epidemiológica de la Dirección General de Epidemiología del Ministerio de Salud.

De lo expuesto en los párrafos precedentes, se concluye que el Estado tiene la regulación normativa necesaria para avocarse al desarrollo de políticas públicas en materia de prevención de propagación de enfermedades internacionales desde al año 2005, fecha de entrada en vigor del RSI.

A continuación, en los párrafos siguientes se procederá a efectuar el análisis de las políticas desarrolladas por el Estado peruano, conforme a las recomendaciones efectuadas tanto por la OMS como por la OPS.

\section{El Estado peruano y la implementación de las políticas públicas en el marco de las recomendaciones de la OMS y de la OPS en materia de prevención de propagación internacional de enfermedades}

\subsection{Creación del Centro Nacional de Enlace y el Centro Nacional de Epidemiología, Prevención y Control de Enfermedades}

El Estado peruano, dando cumplimiento a lo dispuesto en el art. $4^{\circ}$ del RSI, mediante Resolución Ministerial $N^{\circ}$ 793-2006-MINSA, creó el Centro Nacional de Enlace que tuvo como funciones: a) el envío de información urgente a los puntos de contacto de la OMS, b) la difusión y recepción de información epidemiológica a las entidades integrante del Sistema Nacional de Información Epidemiológica.

Con el Centro Nacional de Enlace se inician propiamente las coordinaciones entre el Estado peruano y la OMS. Sin embargo, esta creación no significó el diseño ni mucho menos la aplicación de políticas de salud pública en materia de respuesta ante eventos de propagación internacional de enfermedades. Para este propósito, todavía hacen falta diversas acciones que el Estado tendría que haber implementado, como el diseño de planes y/o programas específicos.

Asimismo, mediante Resolución Ministerial N 793-2006-MINSA se creó el Centro Nacional de Enlace para el Reglamento Sanitario Internacional, siendo este el Centro Nacional de Epidemiología, Prevención y Control de Enfermedades, el cual tiene entre sus funciones velar por el cumplimiento de normas y acuerdos de vigilancia sanitaria internacional establecidos en el RSI.

Este centro tiene como función principal la vigilancia epidemiológica que comprende la aplicación de protocolos desde la etapa de la sospecha, la investigación y, finalmente, la confirmación o es descarte de una enfermedad. Esto permite adoptar las medidas de prevención y control de la enfermedad en la sociedad.

El Centro Nacional de Epidemiología, Prevención y Control de Enfermedades del Ministerio de Salud es el órgano técnico encargado de garantizar una respuesta eficaz y oportuna del Estado en 
materia de prevención de propagación de enfermedades de alcance nacional e internacional. Pero esta función asignada, y este compromiso que debió objetivarse en políticas públicas plasmadas mediante acciones, al parecer no pasa de ser una declaración lírica. La realidad es que hemos experimentado las consecuencias de la precariedad en materia de respuesta ante la propagación del COVID-19. Un Estado sin el control suficiente del sistema sanitario, carente de los más mininos recursos humanos y de equipamiento sanitario, ha provocado la muerte excesiva de ciudadanos.

\subsection{Aprobación del "Plan de Mejoramiento de Capacidades Básicas de la Vigilancia y Respuesta en el País"}

Mediante la Resolución Suprema N 025-2013-SA se creó la "Comisión Multisectorial de naturaleza temporal encargada de elaborar y proponer el Plan de Mejoramiento de Capacidades Básicas de la Vigilancia y Respuesta en el País" -Plan de Mejoramiento-, el cual tuvo como propósito elaborar el plan del mismo nombre que el Estado peruano había omitido desde el año 2007. Por esta razón, el 20 de enero de 2012, se vio en la necesidad de solicitar a la OMS una ampliación del plazo para adecuarse a las exigencias del RSI.

Como puede advertirse, el Estado peruano aprobó recientemente el Plan de Mejoramiento el 18 de junio de 2014. En este se propusieron políticas de vigilancia y respuesta ante la propagación internacional de enfermedades. No se entiende la omisión gubernamental que ocasionó la demora en su aprobación, lo cual impidió también la implementación de acciones concretas de prevención de propagación de enfermedades internacionales. Fueron siete años de retraso, en un mundo cada vez más propenso a la propagación internacional de enfermedades a velocidades inusitadas. Además, si se toma en consideración que las políticas públicas demandan un periodo de implementación relativamente amplio, de todas maneras, esto significaba incrementar los riesgos de una deficiente respuesta ante eventuales eventos de propagación internacional de enfermedades con consecuencias lamentables en la salud de la población.

A través del RSI, la OMS se propuso el objetivo de fortalecer y mantener la capacidad para detectar (vigilar), notificar y responder rápidamente a los eventos de salud pública. Sin embargo, el Estado peruano, mediante este Plan de Mejoramiento, recién creaba el marco normativo para que los sectores involucrados "coordinen y permitan la prevención, control y mitigación de problemas de salud pública con potencialidad de representar una amenaza a nivel nacional y global" (D. S. N 011-2014-SA que aprueba la R. S. No 025-2013; Art. II. Finalidad).

Debe tenerse en consideración, una vez más, lo dispuesto en el artículo 13.1 del RSI. El Perú tenía la obligación de implementar -a más tardar dentro de los 5 años de entrada en vigencia el RSI- las políticas públicas necesarias para reforzar y mantener la capacidad de respuesta pronta y eficaz ante las amenazas y las emergencias de salud pública de importancia internacional. Pero, por distintas razones, entre las que se encuentra la falta de una política coherente en materia de salud pública, la corrupción, el desinterés de los gobiernos, etc., el Estado peruano ha provocado las desgarradoras cifras como país con la más alta tasa de mortalidad en el mundo, como consecuencia del COVID-19 (85.8 por cada 100,000 habitantes). Las consecuencias que hoy sufre la población nacional, sin oxígeno suficiente; sin hospitales de nivel 3 suficientes (con atención especializada) debidamente equipados -léase sin camas $\mathrm{UCl}-$; sin el reforzamiento de la atención primaria (que hubiera permitido mitigar significativamente los efectos de este enfermedad); sin médicos especializados ni enfermeras proporcionales a la demanda poblacional; con escases de medicina o existentes, pero a precios inaccesibles para las mayorías; con una notoria desarticulación entre el servicio privado y el servicio público para la atención de la salud en caso de emergencias, eran totalmente previsibles. 
Un dato de impacto directo en la capacidad de respuesta ante la eventual propagación internacionales de enfermedades es que, hasta el año 2014, el Estado peruano carecía de protocolos básicos para la implementación del RSI. A siete años de vigencia plena del RSI, el Perú había desperdiciado la oportunidad de implementar medidas adecuadas para fortalecer su capacidad de respuesta. Debido a ello, en el numeral 6.4 del Plan de Mejoramiento se detallan las capacidades básicas que no se han logrado y que era necesaria la implementación de actividades concretas para dar cumplimiento a este propósito. Mediante este plan se propone la elaboración del protocolo de coordinación entre el Centro Nacional de Enlace y los órganos que cumplen con las capacidades básicas para la implementación del RSI, la designación del área responsable dentro de la Dirección General de Epidemiología para el control de las acciones en el marco de la implementación del RSI, acondicionamiento de hospitales de nivel 3 en los puntos de entrada al país para pacientes que requieran aislamiento ${ }^{47}$, identificación de necesidad de adquisición de equipos, medicamentos, equipos de protección personal para situaciones de emergencia biológica.

Los hospitales de nivel 3 son aquellos que cuentan con atención especializada, incluido el nivel de atención en cuidados intensivos. El Ministerio de Salud define a este tipo de hospitales como aquellos que están en la posibilidad de garantizar una atención "integral ambulatoria y hospitalaria altamente especializada" (Minsa, 2004 , p. 67). Pero, al parecer, los deficientes implementación y equipamiento de los hospitales tampoco se habrían atendido en su debida oportunidad. Esta problemática -denominada brechas de infraestructura sanitaria en el Perú- ha sido detallado por el Ministerio de Salud, a través de la Oficina de General de Planeamiento, Presupuesto y Modernización, que, en el año 2020, ha publicado el documento denominado "Diagnóstico de Brechas de Infraestructura o accesos a servicios de salud", en el que da cuenta de preocupantes condiciones hospitalarias que, dada la trascendencia, debió dar lugar a su adecuada implementación desde tiempos atrás. 


\begin{tabular}{|c|c|c|c|c|}
\hline DEPARTAMENTO & $\begin{array}{l}\text { HOSPITALES CON CAPACIDAD } \\
\text { INSTALADA INADECUADA }\end{array}$ & $\begin{array}{l}\text { TOTAL DE } \\
\text { HOSPITALES }\end{array}$ & & DICADOR \\
\hline Amazonas & 2 & 7 & $29 \%$ & \\
\hline Ancash & 10 & 12 & $83 \%$ & \\
\hline Apurímac & 4 & 6 & $67 \%$ & \\
\hline Arequipa & 4 & 11 & $36 \%$ & \\
\hline Ayacucho & 6 & 10 & $60 \%$ & \\
\hline Cajamarca & 4 & 11 & $36 \%$ & \\
\hline Callao & 3 & 7 & $43 \%$ & \\
\hline Cusco & 5 & 10 & $50 \%$ & \\
\hline Huancavelica & 3 & 5 & $60 \%$ & \\
\hline Huánuco & 0 & 5 & $0 \%$ & \\
\hline Ica & 0 & 9 & $0 \%$ & \\
\hline Junín & 7 & 12 & $58 \%$ & \\
\hline La Libertad & 15 & 28 & $54 \%$ & \\
\hline Lambayeque & 2 & 6 & $33 \%$ & \\
\hline Lima & 35 & 46 & $76 \%$ & \\
\hline Loreto & 3 & 7 & $43 \%$ & \\
\hline Madre de Dios & 1 & 2 & $50 \%$ & \\
\hline Moquegua & 0 & 4 & $0 \%$ & \\
\hline Pasco & 2 & 6 & $33 \%$ & \\
\hline Piura & 2 & 6 & $33 \%$ & \\
\hline Puno & 9 & 15 & $60 \%$ & \\
\hline San Martín & 3 & 10 & $30 \%$ & \\
\hline Tacna & 0 & 2 & $0 \%$ & \\
\hline Tumbes & 2 & 3 & $67 \%$ & \\
\hline Ucayali & 2 & 3 & $67 \%$ & \\
\hline TOTAL & 124 & 243 & $51 \%$ & \\
\hline \multicolumn{3}{|c|}{$\begin{array}{c}\text { Tabla 1. Indicador porcentaje de hospitales con capacidad instalada ina } \\
\text { decuada (OMPI MINSA RENIPRESS, 2020). }\end{array}$} & & \\
\hline
\end{tabular}

Del cuadro se advierte que, en el Perú, se tienen 243 hospitales de los cuales 124 tienen capacidad instalada inadecuada (precariedad de infraestructura, equipamiento obsoleto, inoperativo u obsoleto). Los más críticos los hospitales de la región Ancash en la que, de 12 hospitales 10, tienen capacidad instalada inadecuada, lo cual constituye un $83 \%$. Lima ocupa el segundo lugar dado que, de 46 hospitales, 35 tienen capacidad instalada inadecuada, lo que constituye un $76 \%$. Esta situación se agrava al considerar que, en el departamento de Lima, se encuentran los dos puntos más importantes de ingreso al país: el Aeropuerto Internacional Jorge Chávez y el Puerto del Callao. En estos existe la mayor probabilidad de propagación internacional de enfermedades y, precisamente, es esta última región donde se ha registrado el mayor índice de mortalidad por COVID-19, alcanzado una cifra de más de 47,000 decesos hasta el 7 de marzo de 2021. 
De las 25 regiones del país, solo 4 regiones (Ica, Tacna, Huánuco y Moquegua) tienen hospitales con capacidad instalada adecuada. De estas, 10 regiones tienen hospitales con capacidad instalada inadecuada entre $50 \%$ a más, lo cual significa que, a nivel nacional, más de la mitad de los hospitales tienen capacidad instalada inadecuada lo que provoca una atención sanitaria deficiente en perjuicio de la salud y la vida de los pacientes.

Conforme a este diagnóstico de brechas de infraestructura en salud, en los centros de primer nivel de atención de salud (atención básica), la capacidad instalada inadecuada alcanza el $77.78 \%$.

Los hospitales de EsSalud también son parte del sistema de atención de la salud a cargo del Estado, en todo el territorio nacional. En ese sentido, los hospitales administrados por esta entidad que brindan servicios en el segundo y tercer nivel de atención suman un total de 73 , de los cuales las redes que corresponden al hospital Almenara y Rebagliati, ambos en la ciudad de Lima, se encuentran entre los establecimiento con mayor capacidad instalada inadecuada (Ministerio de Salud, 2020), junto con las redes de Piura, La Libertad, Lambayeque e Ica, en los que los índices de mortalidad por el COVID-19 han alcanzado las mayores cifras a nivel nacional. Es el caso de Piura (4.79\%), Ica (5.51\%), Lambayeque (5.59\%), La Libertad (6.16\%), Lima Metropolitana (3,19\%), Callao (4,3\%), Lima región (4.38\%) de mortalidad, solo por el COVID-19 (Sala situacional-MINSA).

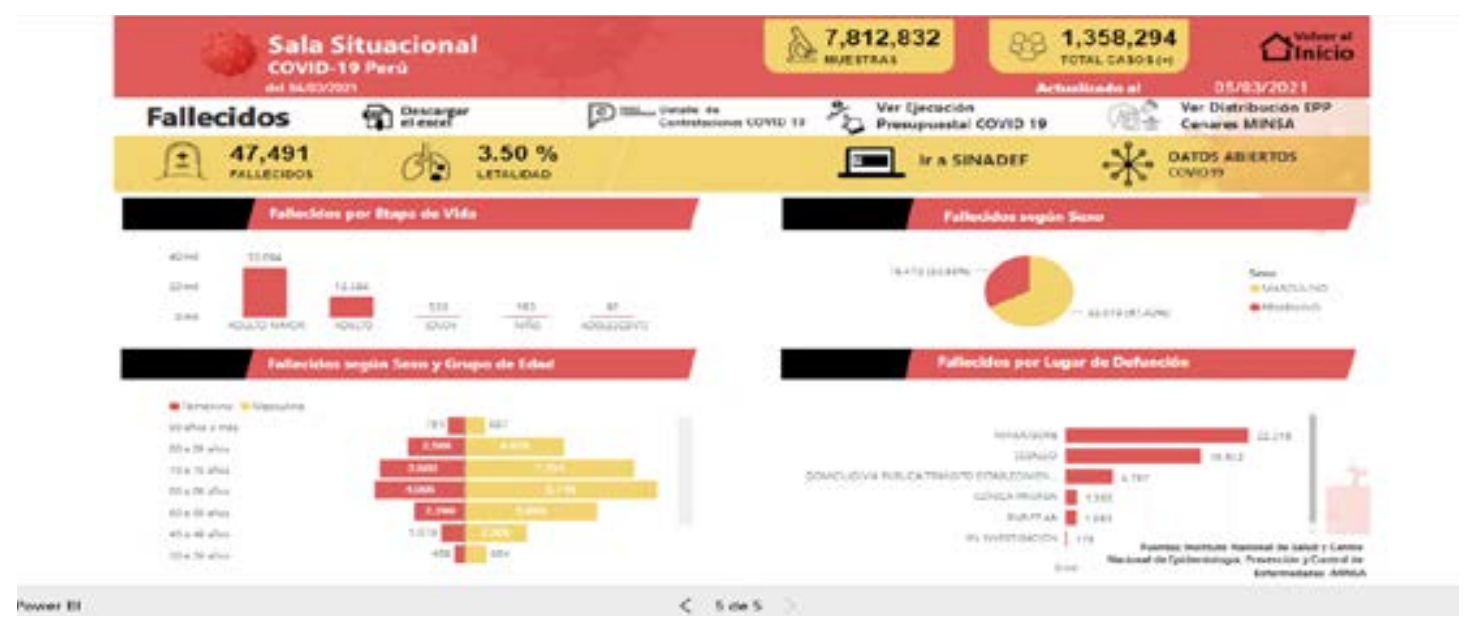

Figura 2. Fallecidos por lugar de defunción en el Perú al 4 de marzo de 2021

Como se advierte, en los hospitales del Ministerio de Salud se han producido el mayor índice de mortalidad por COVID-19 y los hospitales de EsSalud representan el segundo lugar de fallecimientos a nivel nacional. Probablemente, este fenómeno tenga como una de las principales causas a la capacidad instalada inadecuada con los que contaban estos establecimientos antes de la pandemia del COVID-19 y que, a la fecha, es muy probable que esta situación no hay sufrido variaciones sustanciales. Como es el caso del notorio desabastecimiento de oxígeno medicinal y el déficit de camas de cuidados intensivos, indispensable para incrementar la esperanza de sobrevivencia de un paciente infectado por este virus. De marzo de 2020 a marzo de 2021, el Estado no ha podido superar estas deficiencias en la proporción de las necesidades y de la demanda, tanto en la primera como en la segunda ola.

Asimismo, en el marco de la capacidad de respuesta del Estado peruano ante posibles escenarios de propagación de enfermedades como el COVID-19 es importante y sintomático analizar el cuadro siguiente. 


\begin{tabular}{|c|c|c|}
\hline INDICADOR DE BRECHA & $\begin{array}{l}\text { VALOR ABSOLU- } \\
\text { TO }\end{array}$ & PORCENTAJE \\
\hline $\begin{array}{l}\text { 1. Porcentaje de establecimientos de salud } \\
\text { del primer nivel de atención con capacidad } \\
\text { instalada inadecuada. }\end{array}$ & 6785 & $77.78 \%$ \\
\hline $\begin{array}{l}\text { 2. Porcentaje de nuevos establecimientos } \\
\text { de salud requeridos del primer nivel de } \\
\text { atención. }\end{array}$ & 281 & $20.74 \%$ \\
\hline $\begin{array}{l}\text { 3. Porcentaje de hospitales con capacidad } \\
\text { instalada inadecuada. }\end{array}$ & 124 & $51.00 \%$ \\
\hline $\begin{array}{l}\text { 4. Porcentaje de nuevos hospitales requeri- } \\
\text { dos. } \\
\text { 5. Porcentaje de institutos especializados con } \\
\text { capacidad instalada inadecuada }\end{array}$ & 172 & $56.21 \%$ \\
\hline $\begin{array}{l}\text { 6. Porcentaje de laboratorios del Instituto Na- } \\
\text { cional de Salud con capacidad instalada } \\
\text { inadecuada. }\end{array}$ & 9 & $60.00 \%$ \\
\hline $\begin{array}{l}\text { 7. Porcentaje de laboratorios regionales de } \\
\text { salud pública con capacidad instalada ina- } \\
\text { decuada. }\end{array}$ & 39 & $95.12 \%$ \\
\hline $\begin{array}{l}\text { 8. Porcentaje de sedes administrativas con } \\
\text { inadecuado índice de ocupación. }\end{array}$ & 23 & $95.83 \%$ \\
\hline $\begin{array}{l}\text { 9. Porcentaje de sistemas de información de } \\
\text { salud que no funcionan adecuadamente. }\end{array}$ & 1664 & $79.92 \%$ \\
\hline $\begin{array}{l}\text { 10. Porcentaje de centros de promoción y vigi- } \\
\text { lancia comunal requeridos }\end{array}$ & 9 & $75 \%$ \\
\hline & 1264 & $66.91 \%$ \\
\hline
\end{tabular}

Tabla 2. Indicadores de brecha de infraestructura y equipamiento del sector salud 2018 (OPMI-MINSA. 2020)

La OPS, en la Agenda de Salud para las Américas 2008-2017, ya había propuesto en el objetivo 1 garantizar la capacidad de respuesta mediante el desarrollo de la atención médica en el primer nivel de atención. En ese sentido, el Estado peruano debió implementar políticas de desarrollo para el cumplimiento de dicho objetivo. Sin embargo, los actuales establecimientos de salud destinados al primer nivel de atención que existen en el Perú tienen una capacidad instalada inadecuada de $77.78 \%$ del total. Esto quiere decir que, en el Perú, el primer nivel de atención de la salud está prácticamente colapsada y no garantiza una cobertura suficiente ni adecuada.

Un dato alarmante es que en el Perú los laboratorios del Instituto Nacional de Salud prácticamente no sirven. El $95.12 \%$ de los existentes tiene una capacidad instalada inadecuada.

\subsection{El problema de las camas para cuidados intensivos (UCI)}

Un aspecto de vital importancia en el marco de la capacidad de respuesta del Estado peruano frente a la propagación del Covid-19, ha sido el de la capacidad para atender pacientes que requerían atención hospitalaria en casos críticos mediante las camas adecuadas para cuidados intensivos. 
2020), para esta fecha el Perú contaba con una cantidad de 525 camas UCI en todo el país. Esta es una situación crítica sobre la que se han pronunciado diversos especialistas como el Dr. Jesús Valverde, de la Sociedad Peruana de Medicina Intensiva, quien en una entrevista realizada en abril de 2020 sostuvo que la cantidad de camas UCI que tiene el Perú, es una de las más bajas del continente.

\begin{tabular}{|c|c|c|c|c|c|}
\hline \multirow[b]{2}{*}{ Pais 4} & \multicolumn{2}{|c|}{ Nro. camas UCI } & \multicolumn{2}{|c|}{ Nro. camas UCI por 100 mil habitantes } & \multirow[b]{2}{*}{ Nro, de ventiladores mecínicos $\Delta$} \\
\hline & Antes $\mathbf{A}$ & A) $31 / 05 / 2020 \triangle$ & Antes $\mathbf{A}$ & Al $31 / 05 / 20204$ & \\
\hline Argentina & 8.444 & 11.500 & 19,0 & 25,8 & Sin información \\
\hline México & 2.446 & 31.023 & 2,0 & 24,8 & 10.401 \\
\hline Chile & 1.229 & | 2.107 & 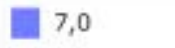 & 12,0 & 3.001 \\
\hline Colombia & Sin informsodón & | 3.289 & Sin informsciọn & 6,8 & 2.817 \\
\hline Perú & 1276 & | 1.573 & 0,9 & I 4,9 & | 1.418 \\
\hline Guatemala & Sin información & | 634 & Sin información & 4,3 & 1676 \\
\hline Bolivia & Sin información & 490 & Sin información & 4,2 & Sin información \\
\hline Ecuador & | 259 & 480 & 1,5 & 12,7 & 1663 \\
\hline El Salvador & 180 & 145 & 1,2 & 12,2 & 1547 \\
\hline Venezuela & 1.213 & Sin información & 3,8 & Sin informacón & Sin información \\
\hline
\end{tabular}

Seguin la Sociedad Peruana de Medicina Intensiva, por cada 100 mil habitantes deberiamos tener por lo menos so camas UCI. Lo mínimo aceptable son 6 camas $\mathrm{UCl}$, pero varios paises de la región estuvieron siempre fuera del rango minimo.

Figura 3. Número de camas UCI por cada 100 mil habitantes en países de América Latina (Sociedad Peruana de Medicina Intensiva).

En efecto, antes del 31 de mayo de 2020, el Perú contaba con 0,9 camas UCI por cada 100 mil habitantes, mientras países como Chile $(7,0)$ o Argentina $(19,0)$ superaban largamente esta cifra. A partir del 31 de mayo, la cantidad de camas UCl se ha incrementado en casi todos los países, pero el nivel de incremento en el caso peruano ha seguido siendo inferior en comparación con los demás países de la región. A partir de esta fecha, el Perú incrementó a 4,9 camas UCI por cada 100 mil habitantes, frente a países como Argentina $(25,8)$, México $(24,8)$, Chile $(12,0)$. Como se puede advertir, estos países superaban significativamente el nivel de equipamiento con camas UCl efectuado por Perú. La Sociedad Peruana de Medicina Intensiva ha declarado que la cantidad aceptable para una adecuada atención de casos críticos es de 10 camas UCI por cada 100 mil habitantes y, en el peor de los casos, 6 camas UCI por cada 100000 habitantes.

En el marco de esta pandemia, según el Presidente de la Sociedad Peruana de Medicina Intensiva, el Dr. Jesús Valverde, al menos $30 \%$ de los pacientes hospitalizados requerirán de una cama UCI. Si tomamos en cuenta que el 11 de marzo de 2021 la cantidad de pacientes hospitalizados ascendía a 14,610, podemos calcular que, de ellos, 4,383 deberán requerir una cama UCl aproximadamente; y sin embargo, el Perú cuenta con solo 2,326 unidades.

En consecuencia, el alto índice de mortalidad por la enfermedad del COVID-19 en el Perú tiene relación directa con el abandono y la carencia de equipamiento adecuado de los hospitales (3.7\% de letalidad). A su vez, este fenómeno tiene una relación indiscutible con el grado de inversión en salud que ha efectuado el Estado peruano. En el año 2000 el Perú invertía en salud, menos del 
$1.5 \%$ y en el año 2014 apenas superaba el $2.0 \%$ del PBI, según el Banco Mundial. Para el año 2020 , el Perú invirtió en salud solo el $3 \%$ del PBI frente a un $8 \%$ del PBI en Cuba y $10 \%$ del PBI en Estados Unidos (Organización Panamericana de la Salud, 2020).

Las muertes por COVID-19 se pudieron mitigar significativamente. Después de las primeras experiencias de marzo de 2020, el Estado pudo prevenir las consecuencias para la segunda ola. Sin embargo, poco o nada se hizo para implementar mejor la infraestructura sanitaria, a sabiendas de los efectos que la población padecería.

\subsection{La política de prevención primordial de la Organización Panamericana de la salud. El caso de la alimentación saludable}

La Organización Panamericana de la Salud (2011) propuso un nivel de prevención denominada "prevención primordial", orientada a evitar el surgimiento de patrones de vida que contribuyan a elevar el riesgo de enfermar, vinculado especialmente con el tipo de alimentación que consume la población, como es el caso de productos altos en grasas o azúcares.

En este marco, el Estado peruano promulgó el 17 de mayo de 2013, la Ley Nㅜ 30021 denominada, Ley de Promoción de Alimentación Saludable para Niños y Adolescente, durante el mandato del expresidente Ollanta Humala. Esta ley estuvo orientada a regular el consumo de productos alto en grasas y azúcares en las instituciones educativas, y su implementación exigía la necesaria coordinación con el Ministerio de Educación. Sin embargo, esta no sería reglamentada hasta el año 2017 mediante D.S. N 017-2017-SA. En el año 2018, mediante Decreto Supremo $N^{\circ} 012-$ 2018-SA, el Poder Ejecutivo aprobó el uso de octógonos para productos que contengan azúcares, grasas saturadas y grasas trans.

Nuevamente, se advierte la aprobación tardía de normas orientadas a la prevención de la salud, la misma que ya era necesaria en el contexto de una creciente tasa de sobrepeso que afecta a la población peruana. Domínguez (2020) ha señalado que el $69.9 \%$ de adultos, $42.4 \%$ de jóvenes, $23.9 \%$ de adolescentes, $32.3 \%$ de escolares y $33.1 \%$ de adultos mayores padecen de sobrepeso y obesidad.

De acuerdo con estudios realizados, existe una tendencia al alza de la obesidad en niños menores de 5 años. En el año 2009, se obtuvo un $6.8 \%$ de sobrepeso y $2.4 \%$ de obesidad (Del Águila, 2017). Esta situación es más crítica en los niños que se encuentran entre los 6 a 9 años, en cuyo caso la tasa de obesidad alcanzó hasta 14.8\% entre los años 2013-2014 (Pajuelo-Ramírez, 2017).

Diseñar políticas que promuevan la alimentación saludable es una necesidad cada vez más urgente. Los datos descritos en el párrafo precedente, no solo advierten una alarmante situación por la alta tasa de obesidad, considerada por la OMS como la epidemia del presente siglo (PajueloRamírez, 2017), sino que esta acarrea el padecimiento de enfermedades conexas coma la diabetes mellitus tipo 2, colesterol, triglicéridos, problemas de articulaciones, asma, hipertensión arterial, el síndrome metabólico, etc., que a la vez constituyen factores que incrementan significativamente el riesgo de muerte frente a la enfermedad del COVID-19.

La lucha contra la obesidad infantil y adolescente requiere la participación conjunta de diversos sectores del Estado y la sociedad, principalmente de ministerios como el de Educación, Salud y de la empresa privada para priorizar la salud pública frente a todo afán lucrativo. Las perspectivas de generar utilidades que tienen las empresas vinculadas a la comercialización de alimentos deben supeditarse a la promoción de la salud y a la prevención de enfermedades.

\subsection{La política pública de aseguramiento universal en salud}

Otra de las propuestas de la Organización Panamericana de la Salud a los Estados Parte, planteada 
en el objetivo 1 de la Agenda de Salud para las Américas 2008-2017, fue el generar condiciones que permitan un acceso universal a la salud y cobertura universal de la salud. En ese contexto, el Estado peruano publicó la Ley $\mathrm{N}^{\circ}$ 29344, Ley Marco de Aseguramiento Universal en Salud, el 9 de abril de 2009, en cuyo artículo $1^{\circ}$ se propuso como finalidad "(...) garantizar el derecho pleno y progresivo de toda persona a la seguridad social en salud (...)". Es decir, con esta norma el Estado se propuso que las personas en todo el territorio nacional gocen de un seguro de salud para acceder a las prestaciones de salud oportunos y eficaces.

En la disposición final se dispuso que, en un plazo de 90 días, el Poder Ejecutivo proceda a reglamentar esta Ley. Sin embargo, el 02 abril de 2010 recién se cumplió con dicha reglamentación mediante la aprobación del Decreto Supremo Nº08-2010-SA.

Según cifras de la Defensoría del Pueblo, para el año 2011 existía un 22.7\% con seguro de EsSalud, 36.1\% con Seguro Integral de Salud (SIS) y 35.5\% de personas no contaban con ningún seguro. Esta cifra se incrementó notoriamente para el año 2019, pues se logró una afiliación de 20 millones de personas. Lamentablemente, no solo se trata de afiliar a la población a un seguro de salud (SIS, EsSalud), sino de brindar una atención de calidad en los centros hospitalarios. Es en este aspecto en el que el Estado peruano no ha desplegado esfuerzos necesarios para prevenir adecuadamente la propagación de enfermedades o para evitar las consecuencias de estas.

\subsection{Capacidades en seguridad sanitaria del Perú}

Según Gozzer, Canchihuamán y Espinoza (2020), la OMS desarrolló un sistema de monitoreo y evaluación de la implementación del RSI sobre la base de modalidades de medición, denominado Informe Anual de Autoevaluación (IAA) del año 2018, y el Índice de Seguridad Sanitaria Global (GHSI) del año 2019. Estos criterios tienen una puntuación de 0 a 100 y permiten clasificar a los países en tres categorías: preparado, medianamente preparado y poco o no preparado.

Conforme a estos índices el Perú se encontraría según el IAA en el puesto 108 de 183 países; pero, en relación al GHSI se encuentra en el puesto 49 de 194. Esto significa que nuestro país se encuentra medianamente preparado para enfrentar pandemias, y que sus principales debilidades radican en la poca preparación en bioseguridad, operaciones de respuestas y emergencias, entre otros aspectos.

Por otro lado, estos mismos autores sostienen que la OMS está implementando "evaluaciones externas conjuntas" orientadas a medir capacidades técnicas, pero que hasta la fecha en el Perú no se ha aplicado. Finalmente, afirman también que en el Perú se advierten "brechas importantes en áreas indispensables para la detección, preparación y respuesta frente a una pandemia" (s/p).

\section{Conclusiones}

El Estado peruano, como Estado parte de la OMS y de la OPS, tiene el deber de acatar las disposiciones normativas emanadas por estos órganos rectores en materia de salud, como es el caso de las disposiciones contenidas en el RSI. Sin embargo, desde el año 2007, fecha que adquirió plena vigencia, el Perú no ha cumplido con implementar adecuadamente las políticas públicas preventivas como respuesta a la propagación internacional de enfermedades, como es el caso del COVID-19, en concordancia con las disposiciones sanitarias internacionales que exigían una actuación oportuna e integral del Estado peruano.

En esa línea, el Estado peruano ha incurrido en graves omisiones frente a las disposiciones de la OMS, en relación al servicio de atención de la salud que viene siendo notoriamente deficiente para enfrentar la grave crisis sanitaria en el contexto de la pandemia lo que ha generado graves consecuencias que pudieron evitarse. Estas omisiones están ligadas a las recomendaciones efectuadas por los organismos rectores de la salud mundial en materia de infraestructura sanitaria, 
aseguramiento universal de la salud y la alimentación saludable.

La omisión sistemática en la asignación de presupuesto suficiente para implementar políticas preventivas y reparadoras en materia de salud, desde las campañas de información sobre el consumo de productos saludables hasta la implementación adecuada de la infraestructura sanitaria, han sido las causas principales de los altos indicies de mortalidad por COVID-19 en el Perú.

1 La Organización Mundial del Comercio fue creada en 1995. En la actualidad agrupa a más de 164 países comprometidos con estas políticas.

2 Conforme a este plan de mejoramiento, los puntos de entrada son Puerto de Paita, Zona frontera con Ecuador, Aeropuerto José Abelardo Quiñones, Puerto Salaverry, Puerto Pisco, Aeropuerto Internacional Jorge Chávez, Puerto del Callao, Puerto de Chimbote y el Puerto de Matarani. 


\section{Referencias}

Breilh, J. (2013) La determinación social de la salud como herramienta de transformación hacia una nueva salud pública (salud colectiva). Revista de la Facultad Nacional de Salud Pública. 31, pp. S13-S27. http://www.scielo.org.co/pdf/rfnsp/v31s1/v31s1a02.pdf

Decreto Supremo N011-2014-MINSA. Recuperado de: https://www.dge.gob.pe/portal/docs/tools/rsi/ DS011_2014_SA_Plan_de_Capacidades_Basicas.pdf

Defensoría del Pueblo (2013). Camino al Aseguramiento Universal en Salud. Resultado de la supervisión nacional a hospitales. Informe Defensorial $N^{\circ} 61$.

Del Águila, V. (2017). Obesidad en el niño: factores de riesgo y estrategias para su prevención en Perú. Revista Peruana de Medicina Experimental y Salud Pública.

Domínguez, C y Lázaro M. (2020). Centro Nacional de Alimentación y Nutrición. Ministerio de Salud.

Garcia Toma. V. (2013). La Constitución Comentada. Comentario al artículo 43 de la Constitución Política del Perú. Editorial Gaceta Jurídica T. I.

Gomez, R. D. (2002). La Noción de "Salud Pública": Consecuencias de la Polisemia. Revista de la Facultad Nacional de Salud Pública., 20 (1).

Gozzer E, Canchihuamán F y Espinoza R. (2020). COVID-19 y la necesidad de actuar para mejorar las capacidades del Perú frente a las pandemias. Rev Peru Med Exp Salud Publica. 37(2), pp. 371373. https://doi.org/10.17843/rpmesp.2020.372.5410.

Junta de Vigilancia Mundial para la Preparación (2019). Un Mundo en Peligro. Informe anual sobre preparación mundial para las emergencias sanitarias. Organización Mundial de la Salud.

Ley General de Salud. [Ley 26842 de 1987]. Recuperado de: http://www.essalud.gob.pe/transparencia/pdf/ publicacion/ley26842.pdf

Ley del Ministerio de Salud. [Ley 27657 de 2002]. Recuperado de: https://cdn.www.gob.pe/uploads/ document/file/283445/255216_L27657.pdf20190110-18386-jxrtyz.pdf

Macinko J, Montenegro H, Nebot Adell C, Etienne C y Grupo de Trabajo de Atención Primaria de Salud de la Organización Panamericana de la Salud (2007). La renovación de la atención primaria de salud en las Américas. Revista Panamericana de la Salud Publica. 21(2/3), pp. 73-84.

Medina, J. (2020). Corrupción en el Sector Privado. Qué la promueve y cómo combatirla. Recuperado de: https://www.proetica.org.pe/noticias/corrupcion-en-el-sector-privado-que-la-promueve-y-comocombatirla/

Ministerio de Salud (2004). Categoría de Establecimiento del Sector Salud. N.T. N 021-MINSA/DGSP. V.01

Ministerio de Salud (2020). Organización del Ministerio de Salud. Recuperado de: https://www.gob.pe/7662ministerio-de-salud-organizacion-de-ministerio-de-salud.

Ministerio de Salud (2006). Resolución Ministerial 793-2006/MINSA. Recuperado de: https://www.dge.gob. pe/portal/docs/tools/rsi/RM793-2006_Creacion_CNE.pdf

Ministerio de Salud (2013). Resolución Suprema N025-2013-SA. Recuperado de: https://www.dge.gob.pe/ portal/docs/tools/rsi/RS025_2013_SA_Comision_Temporal_Plan 